\title{
Analytic calculation of arbitrary matrix elements for the boson exponential quadratic operator
}

\author{
Jian-wei Pan, ${ }^{1}$ Qin-xi Dong, ${ }^{2}$ Yong-de Zhang, ${ }^{3}$ Guang Hou, ${ }^{3}$ and Xiang-bin Wang ${ }^{4}$ \\ ${ }^{1}$ Institut für Experimental Physik, Universität Innsbruck, Technikerstrasse 25, A-6020 Innsbruck, Austria \\ ${ }^{2}$ Gemeinsame Institutseinrichtung Laboratory, Universität Innsbruck, Technikerstrasse 13, A-6020 Innsbruck, Austria \\ ${ }^{3}$ Department of Modern Physics, University of Science and Technology of China, Hefei, Anhui, 230027, People's Republic of China \\ ${ }^{4}$ Department of Physics, Hong Kong University of Science and Technology, Kowloon, Hong Kong, People's Republic of China
}

(Received 9 December 1996)

\begin{abstract}
Making use of the transformation relation between the ordinary form and the antinormal product form of boson exponential quadratic operators (BEQO's), we present an effective method which can be conveniently used to calculate arbitrary matrix elements of BEQO's. By this method, some important matrix elements have been calculated analytically. As a preliminary application, we obtain the exact solution of the density matrix and partition function for the general boson quadratic Hamiltonian without any information for the energy level. As a natural extension, we also obtain the partition function for a general fermion quadratic system. [S1063-651X(97)16208-0]
\end{abstract}

PACS number(s): 05.30. $-\mathrm{d}, 03.65 . \mathrm{Fd}, 42.50 .-\mathrm{p}, 74.20 .-\mathrm{z}$

\section{INTRODUCTION}

It is well known that the calculation of matrix elements for boson exponential quadratic operators (BEQO's) plays a fundamental and important role in modern quantum physics (e.g., the calculation of the density matrix, Feynman's propagator, scattering matrix, etc.). However, to the best of our knowledge, till now only a few special cases have been calculated analytically, and no one has given a universal method to calculate matrix elements of BEQO's.

Recently, Wang and co-workers [1,2] proposed a general approach to transform a BEQO into its normal and antinormal product forms from the viewpoint of linear quantum transformation theory (LQT) [3,4]. Since Refs. [3,4], LQT has been used to solve a series of physical problems $[5,6]$. Motivated by Refs. [2-4], in the present paper we develop a universally effective method to calculate arbitrary matrix elements of BEQO's analytically. By this method, we analytically calculate the transition matrix elements of BEQO's in the particle-number representation, and the matrix elements of BEQO's in the $P$ representation. This method shows that one can obtain analytic expressions of the density matrix and partition function for a general boson quadratic Hamiltonian without any information of the energy level. As a special case, the density matrix and partition functions of coupled nonidentical harmonic osillators (CNHO's) have been briefly discussed. As a natural extension, we also obtain the partition function for a general fermion quadratic system.

\section{GENERAL FORMULA OF CALCULATING MATRIX ELEMENTS OF BEQO's}

Let us introduce the following fundamental operator:

$$
\Lambda=\left(a^{\dagger}, \widetilde{a}\right),
$$

where

$$
\begin{aligned}
a^{\dagger} & =\left(a_{1}^{\dagger}, a_{2}^{\dagger}, \ldots, a_{n}^{\dagger}\right), \\
\tilde{a} & =\left(a_{1}, a_{2}, \ldots, a_{n}\right) .
\end{aligned}
$$

$a_{i}^{\dagger}$ and $a_{i}(i=1,2, \ldots, n)$ are $n$-mode boson creation and annihilation operators, respectively. Thus, without any loss of generality, the ordinary form of a BEQO can be written as [1]

$$
\Omega=\exp \left\{\frac{1}{2} \Lambda N \Sigma_{B} \widetilde{\Lambda}\right\},
$$

where $N$ and $\Sigma_{B} \in C^{2 n \times 2 n}$,

$$
\Sigma_{B}=\left(\begin{array}{rr}
0 & I \\
-I & 0
\end{array}\right),
$$

and $N$ satisfies $\overparen{N \Sigma_{B}}=N \Sigma_{B}$.

Denoting

$$
e^{N}=\left(\begin{array}{cc}
A & D \\
\widetilde{B} & \widetilde{C}
\end{array}\right),
$$

then, by Eq. (5) of Ref. [2] (or see Appendix A), we can obtain the antinormal product expression of $\Omega$,

$$
\begin{aligned}
\Omega & =\exp \left\{\frac{1}{2} \Lambda N \Sigma_{B} \widetilde{\Lambda}\right\} \\
& =[\operatorname{det} A]^{-1 / 2} \ddagger \exp \left\{\frac{1}{2} \Lambda\left(\begin{array}{cc}
-A^{-1} D & 1-A^{-1} \\
1-\widetilde{A^{-1}} & \widetilde{B A}^{-1}
\end{array}\right) \Sigma_{B} \widetilde{\Lambda}\right\} \ddagger
\end{aligned}
$$

where the notation $\ddagger \cdots \ddagger$ means the antinormal product; for example, we have $\ddagger a^{2} a^{\dagger}+a^{\dagger^{2}} a \ddagger=a^{2} a^{\dagger}+a a^{\dagger^{2}}$, while the well-known normal product notation $: \cdots$ : means that $: a^{2} a^{\dagger}+a^{\dagger^{2}} a:=a^{\dagger} a^{2}+a^{\dagger^{2}} a[7]$.

An arbitrary matrix element of the BEQO can be written as

$$
\langle\psi|\Omega| \varphi\rangle .
$$

In Fock space, state vectors $\langle\psi|$ and $|\varphi\rangle$ can be expressed as follows: 


$$
\begin{aligned}
& \langle\psi|=\langle 0| \psi(a), \\
& |\varphi\rangle=\varphi\left(a^{\dagger}\right)|0\rangle,
\end{aligned}
$$

where $\psi(a)\left(\varphi\left(a^{\dagger}\right)\right)$ is a function about $a\left(a^{\dagger}\right)$. Using Eq. (3), we have

$$
\begin{aligned}
&\langle\psi|\Omega| \varphi\rangle=[\operatorname{det} A]^{-1 / 2}\langle 0| \ddagger \psi(a) \\
& \times \exp \left\{\frac{1}{2} \Lambda\left(\begin{array}{cc}
-A^{-1} D & 1-A^{-1} \\
1-\widetilde{A^{-1}} & \widetilde{B A^{-1}}
\end{array}\right) \widetilde{\Lambda}\right\}, \\
& \varphi\left(a^{\dagger}\right) \ddagger|0\rangle .
\end{aligned}
$$

Let us denote

$$
\ddagger \exp \left[a^{\dagger}\left(1-A^{-1}\right) a\right] \ddagger=\sum_{n, m=0}^{\infty} C_{n, m} a^{n} a^{\dagger m} .
$$

Thus Eq. (4) can be rewritten as

$$
\begin{aligned}
\langle\psi|\Omega| \varphi\rangle= & {[\operatorname{det} A]^{-1 / 2}\langle 0| \ddagger \psi(a) e^{(1 / 2) \widetilde{a} B A^{-1} a} } \\
& \times \sum_{n, m=0}^{\infty} C_{n, m} a^{n} a^{\dagger m} e^{-(1 / 2) a^{\dagger} A^{-1} \widetilde{D a^{\dagger}}} \varphi\left(a^{\dagger}\right) \ddagger|0\rangle .
\end{aligned}
$$

Considering the supercomplete multimode coherent state [8]

$$
\int|Z\rangle\langle Z| d^{2} Z=1,
$$

where

$$
\begin{gathered}
\widetilde{Z}=\left(Z_{1}, Z_{2}, \ldots, Z_{n}\right), \quad d^{2} Z=\prod_{i=1}^{n}\left[\frac{d^{2} Z_{i}}{\pi}\right], \\
|Z\rangle=\left|Z_{1}, Z_{2}, \ldots, Z_{n}\right\rangle=\exp \left\{-\frac{1}{2} Z^{\dagger} Z+a^{\dagger} Z\right\}|0\rangle .
\end{gathered}
$$

Inserting Eq. (6) into Eq. (5), we obtain

$$
\begin{aligned}
& \langle\psi|\Omega| \varphi\rangle=\int d^{2} Z[\operatorname{det} A]^{-1 / 2}\left\langle 0\left|\ddagger \psi(a) e^{(1 / 2) \widetilde{a} \widetilde{B} A^{-1} a} \sum_{n, m=0}^{\infty} C_{n, m} a^{n}\right| Z\right\rangle\left\langle Z\left|a^{\dagger^{m}} e^{-(1 / 2) \dot{a}^{\dagger} A^{-1} \widetilde{D a^{\dagger}}} \varphi\left(a^{\dagger}\right) \ddagger\right| 0\right\rangle
\end{aligned}
$$

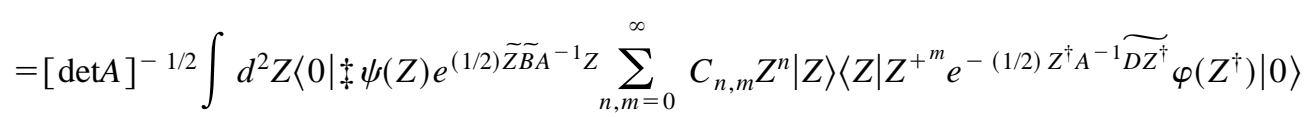

$$
\begin{aligned}
& =[\operatorname{det} A]^{-1 / 2} \int d^{2} Z \psi(Z) \exp \left\{\frac{1}{2}\left(Z, Z^{\dagger}\right)\left(\begin{array}{cc}
-A^{-1} D & -A^{-1} \\
-\widetilde{A}^{-1} & \widetilde{B A^{-1}}
\end{array}\right)\left(\begin{array}{c}
\widetilde{Z^{\dagger}} \\
Z
\end{array}\right)\right\} \varphi\left(Z^{\dagger}\right),
\end{aligned}
$$

where we use the following relation:

$$
\langle 0 \mid Z\rangle=\langle Z \mid 0\rangle=e^{-(1 / 2) Z^{\dagger} Z} .
$$

$$
\varphi\left(a^{\dagger}\right)=e^{\alpha a^{\dagger} \widetilde{a^{\dagger}}+a^{\dagger} \beta}\left(\prod_{j=1}^{n} a_{j}^{+^{n_{J}}}\right),
$$

Now we transform the calculation of the matrix element into a simple integral of the $C$ number. Obviously, the operation of the $C$ number is much more convenient than the operation of the operator. For the convenience of further application, let us consider the following $\psi(a)$ and $\varphi\left(a^{\dagger}\right)$ :

$$
\psi(a)=\left(\prod_{i=1}^{n} a_{i}^{m_{i}}\right) e^{\alpha^{\prime} \tilde{a} a+\widetilde{a} \beta^{\prime}}
$$

where $\alpha, \alpha^{\prime} \in \mathcal{C}, \beta, \beta^{\prime} \in \mathcal{C}^{n}, m_{i}, n_{j}=0,1,2, \ldots$, and

$$
\begin{gathered}
\widetilde{\beta}=\left(\beta_{1}, \beta_{2}, \ldots, \beta_{n}\right), \\
\widetilde{\beta^{\prime}}=\left(\beta_{1}^{\prime}, \beta_{2}^{\prime}, \ldots, \beta_{n}^{\prime}\right) .
\end{gathered}
$$

Then Eq. (7) turns into

$$
\begin{aligned}
\langle\psi|\Omega| \varphi\rangle= & {\left.[\operatorname{det} A]^{-1 / 2} \int\left(\prod_{i=1}^{n} Z_{i}^{m_{i}}\right) e^{\alpha^{\prime} \widetilde{Z} Z+\widetilde{Z} \beta^{\prime}} \exp \left\{\frac{1}{2}\left(Z^{\dagger}, \widetilde{Z}\right)\left(\begin{array}{cc}
-A^{-1} D & -A^{-1} \\
-\widetilde{A^{-1}} & \widetilde{B} A^{-1}
\end{array}\right)\left(\begin{array}{c}
\widetilde{Z^{\dagger}} \\
Z
\end{array}\right)\right\} e^{\alpha Z^{\dagger} \widetilde{Z}^{\dagger}+Z^{\dagger} \beta\left(\prod_{j=1}^{n} Z_{j}^{\dagger_{j}}\right.}\right) d^{2} Z } \\
= & {[\operatorname{det} A]^{-1 / 2}\left[\prod_{i=1}^{n}\left(\frac{d}{d r_{i}}\right)^{m_{i}}\right]\left[\prod_{j=1}^{n}\left(\frac{d}{d s_{j}}\right)^{n_{j}}\right] \int \exp \left\{-\frac{1}{2}\left(Z^{\dagger}, \widetilde{Z}\right)\left(\begin{array}{cc}
-A^{-1} D-2 \alpha & -A^{-1} \\
-\widetilde{A}^{-1} & \widetilde{B} A^{-1}-2 \alpha^{\prime}
\end{array}\right)\left(\begin{array}{c}
\widetilde{Z^{\dagger}} \\
Z
\end{array}\right)+\left(\widetilde{\beta}+\widetilde{s}, \widetilde{\beta^{\prime}}\right.\right.} \\
& \left.+\widetilde{r})\left(\begin{array}{c}
\widetilde{Z^{\dagger}} \\
Z
\end{array}\right)\right\}\left.d^{2} Z\right|_{r=s=0} .
\end{aligned}
$$

Using a Gaussian multiple intergral formula, we have 


$$
\int d^{2} Z \exp \left\{-\frac{1}{2}\left(Z^{\dagger}, \widetilde{Z}\right) Q\left(\begin{array}{c}
\widetilde{Z^{\dagger}} \\
Z
\end{array}\right)+(\widetilde{u}, \widetilde{v})\left(\begin{array}{c}
\widetilde{Z^{\dagger}} \\
Z
\end{array}\right)\right\}=\left[(-1)^{n} \operatorname{det} Q\right]^{-1 / 2} \exp \left\{\frac{1}{2}(\widetilde{u}, \widetilde{v}) Q^{-1}\left(\begin{array}{l}
u \\
v
\end{array}\right)\right\},
$$

where $Q=\widetilde{Q}, Q$ is nonsingular, and $u, v \in \mathcal{C}^{n}$.

Thus we can easily obtain the analytic expression of Eq. (8):

$$
\begin{aligned}
\langle\psi|\Omega| \varphi\rangle= & {\left[\operatorname{det} A \operatorname{det}\left(\begin{array}{cc}
\widetilde{A^{-1}} & -\widetilde{B A^{-1}-2 \alpha^{\prime}} \\
A^{-1} D-2 \alpha & A^{-1}
\end{array}\right)\right]^{-1 / 2}\left[\prod_{i=1}^{n}\left(\frac{d}{d r_{i}}\right)^{m_{i}}\right]\left[\prod_{j=1}^{n}\left(\frac{d}{d s_{j}}\right)^{n_{j}}\right] \exp \left\{\frac{1}{2}\left(\widetilde{\beta}+\widetilde{s,}, \widetilde{\beta}^{\prime}+\widetilde{r}\right)\right.} \\
& \left.\times\left(\begin{array}{cc}
-A^{-1} D-2 \alpha & -A^{-1} \\
-\widetilde{A}^{-1} & \widetilde{B} A^{-1}-2 \alpha^{\prime}
\end{array}\right)^{-1}\left(\begin{array}{c}
\beta+s \\
\beta^{\prime}+r
\end{array}\right)\right\}\left.\right|_{r=s=0} \cdot
\end{aligned}
$$

\section{APPLICATIONS}

In this section, we will calculate the matrix elements of BEQO's in particle-number and coherent-state representations, and obtain the density matrix and partition functions for a general boson quadratic system by making use of Eq. (10).

\section{A. Calculation of $\left\langle m|\Omega| m^{\prime}\right\rangle$ in particle-number representation}

In Fock space, the particle-number eigenstates can be expressed as

$$
\begin{gathered}
\langle m|=\langle 0| \prod_{i=1}^{n} \frac{a_{i}^{m_{i}}}{\sqrt{m_{i} !}}, \\
\left|m^{\prime}\right\rangle=\prod_{j=1}^{n} \frac{a_{j}^{\dagger m_{j}^{\prime}}}{\sqrt{m_{j}^{\prime} !}} .
\end{gathered}
$$

From Eq. (10), we immediately get the transition matrix element

$$
\begin{aligned}
& \left\langle m|\Omega| m^{\prime}\right\rangle \\
& =\left[\operatorname{det} A \operatorname{det}\left(\begin{array}{cc}
\widetilde{A}^{-1} & -\widetilde{B} A^{-1} \\
A^{-1} D & A^{-1}
\end{array}\right)\right]^{-1 / 2} \\
& \times\left[\prod_{i=1}^{n} \frac{1}{\sqrt{m_{i} !}}\left(\frac{d}{d r_{i}}\right)^{m_{i}}\right]\left[\prod_{j=1}^{n} \frac{1}{\sqrt{m_{j}^{\prime} !}}\left(\frac{d}{d s_{j}}\right)^{n_{j}}\right] \\
& \times \exp \left\{\left.\frac{1}{2}\left(\widetilde{s, \widetilde{r})}\left(\begin{array}{cc}
A^{-1} D & A^{-1} \\
\tilde{A}^{-1} & -\widetilde{B} A^{-1}
\end{array}\right)^{-1}\left(\begin{array}{l}
s \\
r
\end{array}\right)\right\}\right|_{r=s=0} .\right.
\end{aligned}
$$

\section{B. Calculation of $\langle-\beta|\Omega| \beta\rangle$ in the coherent-state representation}

$P$ representation is an important application of the coherent state $[9,10]$. In this representation an operator can be written as the following diagonal form:

$$
\Omega=\int\left|Z^{\prime}\right\rangle\left\langle Z^{\prime}\right| P\left(Z^{\prime}\right) d^{2} Z^{\prime}
$$

where

$$
\begin{aligned}
P\left(Z^{\prime}\right)= & \exp \left\{Z^{\prime \dagger} Z\right\} \int d^{2} \beta\langle-\beta|\Omega| \beta\rangle \\
& \times \exp \left\{\beta^{\dagger} \beta+\beta^{\dagger} Z^{\prime}-Z^{\prime \dagger} \beta\right\} .
\end{aligned}
$$

$|\beta\rangle$ is a coherent state. Obviously, the calculation of $\langle-\beta|\Omega| \beta\rangle$ is very important to obtain $P\left(Z^{\prime}\right)$.

For the case of $\langle-\beta|\Omega| \beta\rangle$, we have

$$
\begin{gathered}
\psi(a)=e^{-\left(\beta^{\dagger} \beta / 2\right)-\beta^{\dagger} a}, \\
\varphi\left(a^{\dagger}\right)=e^{-\left(\beta^{\dagger} \beta / 2\right)+a^{\dagger} \beta} .
\end{gathered}
$$

From Eq. (10), we obtain

$$
\begin{aligned}
\langle-\beta|\Omega| \beta\rangle & =\left[\operatorname{det} A \operatorname{det}\left(\begin{array}{cc}
\widetilde{A}^{-1} & -\widetilde{B} A^{-1} \\
A^{-1} D & A^{-1}
\end{array}\right)\right]^{-1 / 2} e^{-\beta^{\dagger} \beta} \\
& \times \exp \left\{\frac{1}{2}\left(\widetilde{\beta},-\beta^{\dagger}\right)\left(\begin{array}{cc}
A^{-1} D & A^{-1} \\
\widetilde{A}^{-1} & -\widetilde{B} A^{-1}
\end{array}\right)\left(\begin{array}{c}
\beta \\
-\widetilde{\beta^{\dagger}}
\end{array}\right)\right\} .
\end{aligned}
$$

\section{Density matrix and partition function for a boson quadratic system}

Let us consider a general quadratic boson system whose Hamiltonian is described by

$$
\hat{H}=a^{\dagger} \alpha a+a^{\dagger} \gamma \widetilde{a}^{\dagger}+\widetilde{a} \gamma^{\dagger} a,
$$

where $\alpha$ is an $n \times n$ Hermitian matrix, and $\gamma$ is an $n \times n$ complex symmetric matrix. If we denote

$$
N=\left(\begin{array}{cc}
\alpha & -2 \gamma \\
2 \gamma^{\dagger} & -\widetilde{\alpha}
\end{array}\right),
$$


Eq. (14) can be rewritten as

$$
\hat{H}=\frac{1}{2} \Lambda N \Sigma_{B} \tilde{\Lambda}-\frac{1}{2} \operatorname{tr} \alpha,
$$

Since $-\frac{1}{2} \operatorname{tr} \alpha$ is a constant, for the convenience of discussion, we could only consider the following Hamiltonian:

$$
\hat{H}=\frac{1}{2} \Lambda N \Sigma_{B} \tilde{\Lambda} .
$$

It is well known that in coordinate representation the densitymatrix elements of the above system are defined as

$$
\rho\left(x, x^{\prime}, \beta\right)=\left\langle x_{1}, x_{2}, \ldots, x_{n}\left|e^{-\beta \hat{H}}\right| x_{1}^{\prime}, x_{2}^{\prime}, \ldots, x_{n}^{\prime}\right\rangle,
$$

where $\beta=1 / k T$, and $k$ is the Boltzmann constant. In Fock space, the coordinate eigenstates can be expressed as

$$
\begin{aligned}
& \left|x^{\prime}\right\rangle=\left|x_{1}^{\prime}, x_{2}^{\prime}, \ldots, x_{n}^{\prime}\right\rangle \\
& =\pi^{-n / 4} e^{-(1 / 2) a^{\dagger} a^{\dagger}+\sqrt{2} a^{\dagger} x^{\prime}-(1 / 2) \widetilde{x^{\prime}} x^{\prime}}|0\rangle, \\
& \langle x|=\left\langle x_{1}, x_{2}, \ldots, x_{n}\right|=\pi^{-n / 4} e^{-(1 / 2) \widetilde{a} a+\sqrt{2} \widetilde{a} x-(1 / 2) \widetilde{x} x} .
\end{aligned}
$$

Denoting

$$
e^{-\beta N}=\left(\begin{array}{cc}
A_{1} & D_{1} \\
\widetilde{B_{1}} & \widetilde{C_{1}}
\end{array}\right),
$$

then, from Eq. (10), we finally obtain the analytic expression of the density matrix elements

$$
\begin{aligned}
& \left\langle x\left|e^{-\beta \hat{H}}\right| x^{\prime}\right\rangle \\
& =\pi^{-n / 2} e^{-(1 / 2)\left(\widetilde{x^{\prime}} x^{\prime}+\widetilde{x x}\right)} \\
& \times\left[\operatorname{det} A_{1} \operatorname{det}\left(\begin{array}{cc}
{\widetilde{A_{1}}}^{-1} & -{\widetilde{B_{1}}}^{-1}{ }^{-1}+1 \\
A_{1}{ }^{-1} D_{1}+1 & A_{1}{ }^{-1}
\end{array}\right)\right]^{-1 / 2} \\
& \times \exp \left\{\left(\widetilde{x^{\prime}}, \widetilde{x}\right)\left(\begin{array}{cc}
A_{1}{ }^{-1} D_{1}+1 & A_{1}^{-1} \\
{\widetilde{A_{1}}}^{-1} & -{\widetilde{B_{1}}}^{-1}{ }^{-1}+1
\end{array}\right)^{-1}\left(\begin{array}{c}
x^{\prime} \\
x
\end{array}\right)\right\} \text {. }
\end{aligned}
$$

Blainzot obtained the exact solution of the partition function for the Hamiltonian $\hat{H}_{0}=\Sigma_{i j} a_{i}^{\dagger} h_{i j} a_{j}[11]$ :

$$
\operatorname{Tr}\left(e^{-\beta \hat{H}_{0}}\right)=\operatorname{det}\left(1-e^{-\beta h}\right)^{-1} .
$$

Equation (19) is very important in the Hartree-Fock approximation of the partition function, because one can always use it to approximate the partition function of the quadratic Hamiltonian $\hat{H}^{\prime}$,

$$
\hat{H}^{\prime}=\sum_{i j} T_{i j} a_{i}^{\dagger} a_{j}+\frac{1}{4} \sum_{i j k l} V_{i j k l} a_{i}^{\dagger} a_{j} a_{k}^{\dagger} a_{l} .
$$

However, a more complete quadratic Hamiltonian could be described by Eq. (16). To our knowledge, no one has ever obtained the exact solution of the partition function for the Hamiltonian $\hat{H}$.

Using Eq. (18), it is easily to obtain the partition function for the Hamiltonian $\hat{H}$ (see Appendix B),

$$
\begin{aligned}
\operatorname{Tr}\left(e^{-\beta \hat{H}}\right)= & \left.\int d x\left\langle x\left|e^{-\beta \hat{H}}\right| x^{\prime}\right\rangle\right|_{x=x^{\prime}} \\
= & \pi^{-n / 2}\left[\operatorname{det} A_{1} \operatorname{det}\left(\begin{array}{cc}
{\widetilde{A_{1}}}^{-1} & -\widetilde{B_{1} A_{1}}{ }^{-1}+1 \\
A_{1}^{-1} D_{1}+1 & A_{1}^{-1}
\end{array}\right)\right]^{-1 / 2} \\
& \times \int d x \exp \left\{\left(\widetilde{x, \widetilde{x})}\left(\begin{array}{cc}
A_{1}^{-1} D_{1}+1 & A_{1}^{-1} \\
{\widetilde{A_{1}}}^{-1} & -{\widetilde{B_{1}}}^{-1}+1
\end{array}\right)^{-1}\left(\begin{array}{l}
x \\
x
\end{array}\right)-\widetilde{x x}\right\}\right. \\
= & \left|\operatorname{det} A_{1} \operatorname{det}\left(\begin{array}{cc}
A_{1}^{-1} D_{1} & A_{1}^{-1}-1 \\
{\widetilde{A_{1}}}^{-1}-1 & -\widetilde{B}_{1} A_{1}^{-1}
\end{array}\right)\right|^{-1 / 2} .
\end{aligned}
$$

Furthermore, Eq. (20) can be simplified as (also see Appen$\operatorname{dix} B$ )

$$
\operatorname{Tr}\left(e^{-\beta \hat{H}}\right)=\left|\operatorname{det}\left(e^{\beta N}-1\right)\right|^{-1 / 2} .
$$

Up to now, we have first obtained exact solutions of the density matrix and partition function for a boson quadratic system. It is easy to verify that the result of Eq. (21) is consistent with that of Blainzot. We would like to emphasize the following: Usually, the partition function can be evaluated only after we have some knowledge about the energy levels of the system. But for the quadratic system we can now evaluate its partition function without any knowledge about its energy levels by using the antinormal product technique. We also note that Bogolubov [12] presented a program to solve the energy levels of the quadratic system, but the analytic solution of the density matrix and partition function for a boson quadratic system has never been given, to our knowledge. Indeed, our method at least has two advantages: First, from the theoretical point of view, using Eq. (21) one can directly discuss some global features of the system. Second, from the point of view of practical computation, Eq. (21) can greatly reduce computation time and simplify the computation program, because in Eq. (21) the crucial computation step only involves the computation of $(\beta N)^{n} / n$ !, and in the sense of approximation one can interrupt the com- 
putation of the computer at some suitable number $n$ after arriving at the desired accuracy.

In a recent paper [13], Fan and Chen obtained the solution of the density matrix for a triatomic linear molecule which is a type of three-dimensional CNHO. However, in the literature the solution for an arbitrary number of CNHO is still left as a challenge for the future $[13,14]$. By using Eqs. (18) and (21), we can directly obtain the density matrix and the partition function for an arbitrary $\mathrm{CNHO}$, whose Hamiltonian is given by

$$
\begin{aligned}
\hat{H} & =\sum_{i=1}^{n}\left[\frac{\hat{P}_{i}^{2}}{2 m_{i}}+\frac{1}{2} m_{i} \omega_{i}{ }^{2} \hat{x}_{i}{ }^{2}\right]+\frac{1}{2} \sum_{i, j=1}^{n} k_{i j} \hat{x}_{i} \hat{x}_{j} \\
& =\tilde{\hat{P}}(2 M)^{-1} \hat{P}+\tilde{\hat{x}}\left(\frac{1}{2} M \Omega^{2}+\frac{1}{2} K\right) \hat{x},
\end{aligned}
$$

where

$$
\begin{aligned}
& \widetilde{\hat{P}}=\left(\hat{P}_{1}, \hat{P}_{2}, \ldots, \hat{P}_{n}\right), \quad \tilde{\hat{x}}=\left(\hat{x}_{1}, \hat{x}_{2}, \ldots, \hat{x}_{n}\right), \\
& M=\left(\begin{array}{cccc}
m_{1} & & & 0 \\
& m_{2} & & \\
& & \ddots & \\
& 0 & & m_{n}
\end{array}\right) \\
& \Omega=\left(\begin{array}{cccc}
\omega_{1} & & & 0 \\
& \omega_{2} & & \\
& & \ddots & \\
& 0 & & \omega_{n}
\end{array}\right), \quad K=\left(K_{i j}\right)_{n \times n} .
\end{aligned}
$$

Defining

$$
(\tilde{\hat{x}}, \widetilde{\hat{P}})=\left(a^{\dagger}, \widetilde{a}\right)\left(\begin{array}{cc}
\frac{1}{\sqrt{2}} & i \frac{\hbar}{\sqrt{2}} \\
\frac{1}{\sqrt{2}} & -i \frac{\hbar}{\sqrt{2}}
\end{array}\right)
$$

we easily have

$$
\begin{gathered}
{\left[a_{i}^{\dagger}, a_{j}\right]=\delta_{i j},} \\
|x\rangle=\pi^{-n / 4} e^{-(1 / 2) a^{\dagger} a^{\dagger}+\sqrt{2} a^{\dagger} x-(1 / 2) \widetilde{x} x}|0\rangle,
\end{gathered}
$$

where $x$ is a $C$ number, thus Eq. (22) reads

$$
\begin{aligned}
\hat{H} & =\frac{1}{2}\left(a^{\dagger}, \widetilde{a}\right) \\
& \times\left(\begin{array}{cc}
\frac{1}{2} M \Omega^{2}+\frac{1}{2} K-\frac{\hbar^{2}}{2 M} & \frac{1}{2} M \Omega^{2}+\frac{1}{2} K+\frac{\hbar^{2}}{2 M} \\
\frac{1}{2} M \Omega^{2}+\frac{1}{2} K+\frac{\hbar^{2}}{2 M} & \frac{1}{2} M \Omega^{2}+\frac{1}{2} K-\frac{\hbar^{2}}{2 M}
\end{array}\right)\left(\begin{array}{c}
\widetilde{a^{\dagger}} \\
a
\end{array}\right) \\
& =\frac{1}{2} \Lambda N \Sigma_{B} \widetilde{\Lambda},
\end{aligned}
$$

where

$$
N=\left(\begin{array}{cc}
\frac{1}{2} M \Omega^{2}+\frac{1}{2} K-\frac{\hbar^{2}}{2 M} & \frac{1}{2} M \Omega^{2}+\frac{1}{2} K+\frac{\hbar^{2}}{2 M} \\
\frac{1}{2} M \Omega^{2}+\frac{1}{2} K+\frac{\hbar^{2}}{2 M} & \frac{1}{2} M \Omega^{2}+\frac{1}{2} K-\frac{\hbar^{2}}{2 M}
\end{array}\right) .
$$

Let

$$
e^{-\beta N}=\left(\begin{array}{ll}
A_{2} & D_{2} \\
\widetilde{B_{2}} & \widetilde{C_{2}}
\end{array}\right) .
$$

Thus, from Eqs. (18) and (21), one can directly obtain the exact solution of the density matrix and partition function for Eq. (22).

\section{Case of the fermion quadratic system}

As a natural extension, let us consider the general fermion quadratic Hamiltonian as follows:

$$
\hat{H}=b^{\dagger} \alpha b+b^{\dagger} \gamma \widetilde{b^{\dagger}}+\widetilde{b} \gamma^{\dagger} b
$$

where $\alpha$ is an $n \times n$ Hermitian matrix, $\gamma$ is an $n \times n$ antisymmetry complex matrix, and

$$
b^{\dagger}=\left(b_{1}^{\dagger}, b_{2}^{\dagger}, \ldots, b_{n}^{\dagger}\right)
$$

$$
\widetilde{b}=\left(b_{1}, b_{2}, \ldots, b_{n}\right) .
$$

$b_{i}^{\dagger}$ and $b_{i}(i=1,2, \ldots, n)$, respectively, are fermion creation and annihilation operators in $n$-mode Fock space. Equation (23) can be rewritten as

$$
\hat{H}=\frac{1}{2} \Lambda N \Lambda^{\dagger}+\frac{1}{2} \operatorname{tr} \alpha
$$

where

$$
\Lambda=\left(b^{\dagger}, \tilde{b}\right) \quad N=\left(\begin{array}{cc}
\alpha & 2 \gamma \\
2 \gamma^{\dagger} & -\widetilde{\alpha}
\end{array}\right)
$$

Now we would like to point out that one can also easily construct the antinormal product technique for the fermion case within the frame of the Grassmann number [8]. Thus after a similar deduction we can finally obtain the partition function for the fermion quadratic system: 


$$
Z(\beta)=e^{-(1 / 2) \beta \operatorname{tr} \alpha}\left|\operatorname{det}\left(e^{\beta N}+1\right)\right|^{1 / 2} .
$$

\section{ACKNOWLEDGMENTS}

The authors acknowledge fruitful discussions with $\mathrm{Z}$. Tang (Atlanta) and S. X. Yu (Vienna). J.W.P. and Q.X.D. were partly supported by the OAD service, Y.D.Z. was supported by the NSF (China) and FWF (Austria).

\section{APPENDIX A: PROOF OF EQ. (3)}

Indeed, operator $\Omega$ can be regarded as a linear quantum transformation (LQT) operator. Let us denote

$$
M=e^{N}=\left(\begin{array}{cc}
A & D \\
\widetilde{B} & \widetilde{C}
\end{array}\right) .
$$

By Ref. [4], $\Omega$ and $M$ thus satisfy

$$
\begin{gathered}
\Omega \Lambda \Omega^{-1}=\Lambda M, \\
M \Sigma_{B} \widetilde{M}=\widetilde{M} \Sigma_{B} M=\Sigma_{B} .
\end{gathered}
$$

Using Eq. (A2), we immediately have

$$
\begin{aligned}
& \widetilde{A B}-B A=0, \\
& \widetilde{A} \widetilde{C}-B D=1 .
\end{aligned}
$$

If $A^{-1}$ exists, by Eq. (A3) one can obtain

$$
\widetilde{C}=\widetilde{A}^{-1}+\widetilde{B A}{ }^{-1} D,
$$

by which matrix $M$ can thus be rewritten as

$$
M=e^{N}=\left(\begin{array}{cc}
1 & 0 \\
\widetilde{B} A^{-1} & -1
\end{array}\right)\left(\begin{array}{cc}
A & 0 \\
0 & \widetilde{A}^{-1}
\end{array}\right)\left(\begin{array}{cc}
1 & A^{-1} D \\
0 & 1
\end{array}\right) .
$$

Therefore, the action of $\Omega$ is equivalent to the product of three LQT operators, i.e.,

$$
\Omega \Leftrightarrow \Omega_{+} \Omega_{0} \Omega_{-},
$$

where $\Omega_{+}, \Omega_{0}$, and $\Omega_{-}$, respectively, satisfy

$$
\begin{gathered}
\Omega_{+} \Lambda \Omega_{+}^{-1}=\Lambda\left(\begin{array}{cc}
1 & 0 \\
\widetilde{B A} A^{-1} & 0
\end{array}\right), \\
\Omega_{0} \Lambda \Omega_{0}^{-1}=\Lambda\left(\begin{array}{cc}
A & 0 \\
0 & \widetilde{A}^{-1}
\end{array}\right), \\
\Omega_{-} \Lambda \Omega_{-}^{-1}=\Lambda\left(\begin{array}{cc}
1 & A^{-1} D \\
0 & 1
\end{array}\right) .
\end{gathered}
$$

From Eq. (20) of Ref. [3], we can easily obtain

$$
\begin{gathered}
\Omega_{+}=\exp \left\{\frac{1}{2} \Lambda N_{+} \Sigma_{B} \widetilde{\Lambda}\right\}, \\
\Omega_{0}=\exp \left\{\frac{1}{2} \Lambda N_{0} \Sigma_{B} \widetilde{\Lambda}\right\},
\end{gathered}
$$

$$
\Omega_{-}=\exp \left\{\frac{1}{2} \Lambda N_{-} \Sigma_{B} \widetilde{\Lambda}\right\},
$$

where

$$
\begin{gathered}
N_{+}=\left(\begin{array}{cc}
0 & 0 \\
\widetilde{B} A^{-1} & 0
\end{array}\right), \quad N_{0}=\left(\begin{array}{cc}
\ln A & 0 \\
0 & \ln A^{-1}
\end{array}\right), \\
N_{-}=\left(\begin{array}{cc}
0 & A^{-1} D \\
0 & 0
\end{array}\right) .
\end{gathered}
$$

Because $\Omega_{+}, \Omega_{0}$, and $\Omega_{-}$, respectively, only contain terms of $a_{i} a_{j}, a_{i}^{\dagger} a_{j}$, and $a_{i}^{\dagger} a_{j}^{\dagger}$, using the following formula [7]:

$$
e^{a^{\dagger} \ln A a+\operatorname{tr} A}=\ddagger e^{a^{\dagger}\left(1-A^{-1}\right) a_{\ddagger}}
$$

and Eqs. (A6)-(A9), one can then obtain the antinormal product expression of $\Omega$ :

$$
\begin{aligned}
\Omega_{A N} & =\Omega_{+} \Omega_{0} \Omega_{-} \\
& =\ddagger \exp \left\{\frac{1}{2} \Lambda\left(\begin{array}{cc}
-A^{-1} D & 1-A^{-1} \\
1-\widetilde{A^{-1}} & \widetilde{B} A^{-1}
\end{array}\right) \Sigma_{B} \widetilde{\Lambda}\right\} \ddagger
\end{aligned}
$$

Obviously $\Omega_{\mathrm{AN}}$ performs the same LQT, i.e,

$$
\Omega_{\mathrm{AN}} \Lambda \Omega_{\mathrm{AN}}^{-1}=\Lambda M .
$$

Thus by Ref. [4], we have

$$
\Omega=\text { const } \times \Omega_{\mathrm{AN}},
$$

where const is a $C$ number. Imitating the calculation of the Appendix of Ref. [1], it is easy to calculate the expectation values of $\Omega$ and $\Omega_{\mathrm{AN}}$ in the vacuum state:

$$
\langle 0|\Omega| 0\rangle=[\operatorname{det} C]^{-1 / 2},
$$

$$
\left\langle 0\left|\Omega_{\mathrm{AN}}\right| 0\right\rangle=[\operatorname{det} A / \operatorname{det} C]^{-1 / 2} .
$$

Finally, we arrive at Eq. (3),

$$
\Omega=[\operatorname{det} A]^{-1 / 2} \Omega_{\mathrm{AN}} .
$$

APPENDIX B: DEDUCTION OF EQS. (20) AND (21)

Proof 1: Denoting

$$
\left(\begin{array}{cc}
A_{1}{ }^{-1} D_{1}+1 & A_{1}^{-1} \\
{\widetilde{A_{1}}}^{-1} & -{\widetilde{B_{1}}}^{-1}{ }^{-1}+1
\end{array}\right)^{-1}=\left(\begin{array}{cc}
T & W \\
U & V
\end{array}\right),
$$

we have 
$\operatorname{Tr}\left(e^{-\beta \hat{H}}\right)$

$$
\begin{aligned}
& =\pi^{-n / 2}\left[(-1)^{n} \operatorname{det} A_{1} \operatorname{det}\left(\begin{array}{ll}
T & W \\
U & V
\end{array}\right)^{-1}\right]^{-1 / 2} \\
& \times \int d x \exp \{\widetilde{x}(T+W+U+V-1) x\} \\
& =\left[(-1)^{n} \operatorname{det} A_{1} \operatorname{det}\left(\begin{array}{ll}
T & W \\
U & V
\end{array}\right)^{-1}\right. \\
& \left.\times \operatorname{det}\left(\begin{array}{cc}
1-T-W & -T-W \\
-U-V & 1-U-V
\end{array}\right)\right]^{-1 / 2} \\
& =\left\{( - 1 ) ^ { n } \operatorname { d e t } A _ { 1 } \operatorname { d e t } \left[\left(\begin{array}{cc}
T & W \\
U & V
\end{array}\right)^{-1}+\left(\begin{array}{cc}
-1 & 0 \\
0 & -1
\end{array}\right)\right.\right. \\
& \left.\left.+\left(\begin{array}{cc}
0 & -1 \\
-1 & 0
\end{array}\right)\right]\right\}^{-1 / 2} \\
& =\left[(-1)^{n} \operatorname{det} A_{1} \operatorname{det}\left(\begin{array}{ll}
A_{1}{ }^{-1} D_{1} & A_{1}{ }^{-1}-1 \\
{\widetilde{A_{1}}}^{-1}-1 & -\widetilde{B}_{1} A_{1}{ }^{-1}
\end{array}\right)\right]^{-1 / 2} \\
& =\left|\operatorname{det} A_{1} \operatorname{det}\left(\begin{array}{ll}
A_{1}{ }^{-1} D_{1} & A_{1}{ }^{-1}-1 \\
\widetilde{A}_{1}^{-1}-1 & -\widetilde{B}_{1} A_{1}{ }^{-1}
\end{array}\right)\right|^{-1 / 2} \text {. }
\end{aligned}
$$

Proof 2: Because $N$ satisfies

$$
\widetilde{N \Sigma_{B}}=N \Sigma_{B},
$$

by Ref. [4], one easily finds

$$
e^{-\beta N} \Sigma_{B} e^{-\widetilde{\beta N}}=\Sigma_{B}
$$

and

$$
C_{1}=A_{1}{ }^{-1}+\widetilde{D_{1} \widetilde{A}_{1}}{ }^{-1} B_{1} .
$$

Therefore,

$$
e^{\beta N}=\Sigma_{B} e^{-\widetilde{\beta N} \Sigma_{B}}{ }^{-1}=\left(\begin{array}{cc}
C_{1} & -\widetilde{D_{1}} \\
-B_{1} & \widetilde{A_{1}}
\end{array}\right)
$$

Thus

$$
\begin{aligned}
& \left|\operatorname{det}\left(e^{\beta N}-1\right)\right|=\left|\operatorname{det}\left(\begin{array}{cc}
C_{1}-1 & -\widetilde{D_{1}} \\
-B_{1} & \widetilde{A_{1}}-1
\end{array}\right)\right| \\
& =\mid \operatorname{det}\left(\begin{array}{cc}
A_{1}{ }^{-1}+\widetilde{D_{1}}{\widetilde{A_{1}}}^{-1} B_{1}-1 & -\widetilde{D_{1}} \\
-B_{1} & \widetilde{A_{1}}-1
\end{array} \mid\right. \\
& =\left|\operatorname{det}\left(\begin{array}{cc}
A_{1}{ }^{-1}-1 & -\widetilde{D} \widetilde{A_{1}}-1 \\
-B_{1} & \widetilde{A_{1}}-1
\end{array}\right)\right| \\
& =\left|\operatorname{det}\left(\begin{array}{cc}
-\widetilde{D_{1}}{\widetilde{A_{1}}}^{-1} & A_{1}^{-1}-1 \\
\widetilde{A_{1}}-1 & -B_{1}
\end{array}\right)\right| \\
& =\left|\operatorname{det}\left(\begin{array}{cc}
\widetilde{D_{1} \widetilde{A}_{1}^{-1}} & A_{1}{ }^{-1}-1 \\
1-\widetilde{A_{1}} & -B_{1}
\end{array}\right)\right| \\
& =\left|\operatorname{det} \widetilde{A_{1}}\right|\left|\operatorname{det}\left(\begin{array}{cc}
A_{1}{ }^{-1} D_{1} & A_{1}{ }^{-1}-1 \\
\widetilde{A_{1}}-1-1 & -\widetilde{B_{1}} A_{1}{ }^{-1}
\end{array}\right)\right| \text {. }
\end{aligned}
$$

[1] X. B. Wang, S. X. Yu, and Y. D. Zhang, J. Phys. A 27, 6563 (1994).

[2] X. B. Wang, Y. D. Zhang, and J. W. Pan, Chin. Phys. Lett. 13, 401 (1996).

[3] R. Balian and E. Brezin, Nuovo Cimento 64, 37 (1969).

[4] Y. D. Zhang and Z. Tang, J. Math. Phys. (N.Y.) 34, 5369 (1993); Nuovo Cimento B 109, 387 (1994).

[5] Y. D. Zhang, G. Badurek, H. Rauch, and J. Summhammer, Phys. Lett. A 188, 225 (1994).

[6] S. X. Yu, H. Rauch, and Y. D. Zhang, Phys. Rev. A 52, 2585 (1995); Z. Tang, ibid. 52, 3448 (1995).

[7] For a detailed definition of the antinormal product, refer to $\mathrm{H}$. Y. Fan, J. Phys. A 25, 1013 (1992), and references therein.
[8] J. R. Klauder and B. S. Skagerstam, Applications in Physics and Mathematical Physics (World Scientific, Singapore, 1986).

[9] R. J. Glauber, Phys. Rev. Lett. 10, 84 (1963).

[10] E. C. G. Sudarshan, Phys. Rev. Lett. 10, 277 (1963).

[11] J. P. Blainzot, Quantum Theory of Finite Systems (MIT Press, Cambridge, MA, 1986).

[12] N. N. Bogolubov, Introduction to Quantum Statistics (High Education Press, Beijing, 1994) (in Chinese).

[13] H. Y. Fan and B. Z. Chen, Phys. Rev. A 50, 3754 (1994).

[14] F. Michelot, Phys. Rev. A 45, 4271 (1992); H. Y. Fan, ibid. 47, 2379 (1993). 